\title{
A note on long-term recognition memory for pictorial material'
}

RAYMOND S. NICKERSON, BOLT BERANEK AND NEWMAN INC. 50 MOULTON ST., Cambridge, Mass. 02138

In an earlier experiment Ss examined an extended sequence of photographs attempting to identify those photos that were occurring for the second time within the sequence. This note describes the results obtained when, after periods ranging from a day to a year, the same $S s$ were asked to distinguish between new photos and photos that they had seen while participating in the original experiment.

A previous report described the results of an experiment in which S's task was to inspect an extended sequence of photographs of assorted content and identify those which were occurring for the second time within the sequence. It was noted that the probability of recognizing the recurrence of a photo as such was very high even when as many as 200 items intervened between its first and second occurrence (Nickerson, 1965).

This note describes an extension of the earlier study. After periods of time ranging from a day to a year following their participation in the original experiment, the same Ss were asked to inspect an additional set of photos, identifying those which had been included in the set they had examined during the original experiment.

Method. Stimuli were 200 black and white photographs, of miscellaneous content, selected from photography periodicals. One hundred of the photos were duplicates of those used in the prior experiment, i.e., "old," and 100 were unique to this study, i.e., "new." Of the 100 old photos, approximately half had occurred twice, and the remainder only once, during Experiment 1. The $S$ examined the photos at the rate of one about every $5 \mathrm{sec}$, turning the pages of a notebook in time with an audio signal, and announcing either "old" or "new" with respect to each photo.

The Ss who participated in Experiment 1 were divided into four groups, the interexperimental interval being 1, 7,28 and approximately 360 days for Groups 1 through 4 , respectively. The Ss were not informed of the intention to conduct Experiment 2 until after they had participated in Experiment 1. (The fact is the intention did not exist until Experiment 1 was well under way. The idea for Experiment 2 was strictly an afterthought, motivated by the offlhand assumption that such data might be of interest to students of memory whatever their theoretical predilections.)

Results and Discussion. Figure 1 shows the performance measures of interest (probabilities estimated from obtained relative frequencies) as functions of the duration of the interexperiment interval, $p\left(R_{o} \mid S_{o}\right)$ and $p\left(R_{o} \mid S_{n}\right)$ representing respectively the probability of the response "old" given an old stimulus and the probability of the response "old" given a new stimulus. The subscript on $S_{o}$ indicates whether the stimulus occurred once or twice in the original experiment.

The probability of a correct response, $p(C R)$, dropped from .92 to .63 as the interval increased from 1 to 360 days. Although exactly half of the photos were old, the probability of the response "new" was consistently greater than that of the response "old," and increased regularly from approximately .56 to about .70 over the range of intervals used. As a consequence of this growing response bias, the "false alarm" rate, i.e., the probability of responding "old" to a new stimulus, remained relatively low throughout the experiment, reaching its highest value, .17, with an interval of 360 days. It follows, of course, that the probability of correctly identifying a new stimulus as such, $1-p\left(R_{o} \mid S_{n}\right)$, was consistently high.

It is clear from Fig. 1 that the likelihood that an old photo would be recognized as such was considerably greater if it had occurred twice during the original experiment. Moreover, the difference between $p\left(R_{0} \mid S_{02}\right)$ and $p\left(R_{0} \mid S_{01}\right)$ remained relatively constant over intervals. For only one of the $S s$ was $p\left(R_{0} \mid S_{0]}\right)$ greater than $\mathrm{p}\left(\mathrm{R}_{\mathrm{O}} \mid \mathrm{S}_{\mathrm{O}}\right)$, and in this case the two scores were nearly equal.

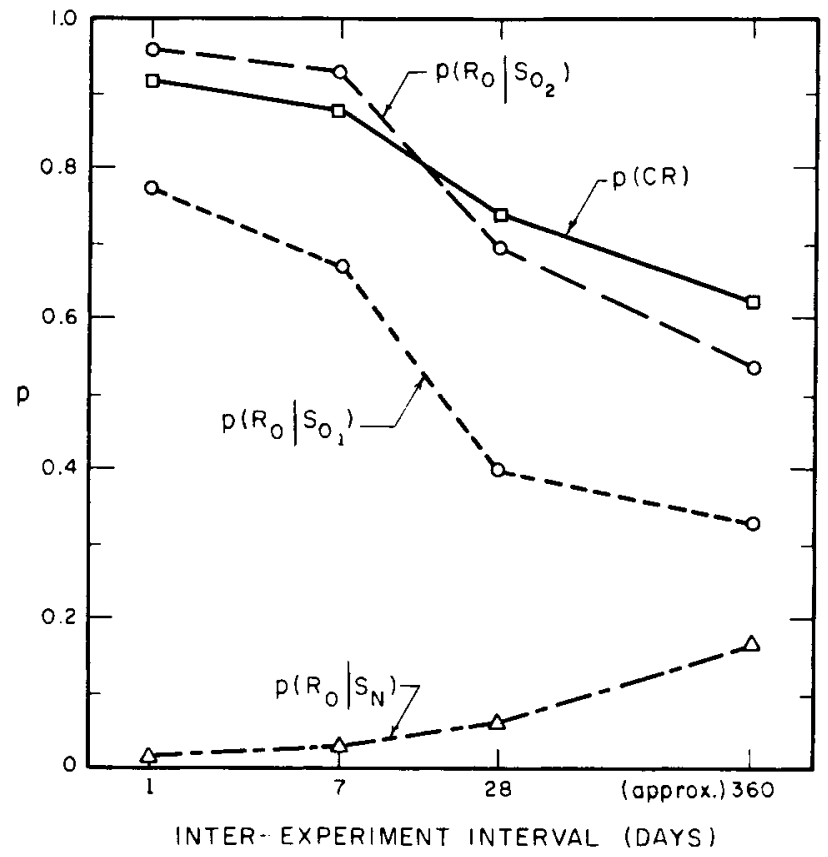

Fig. 1. The probabilities of interest as functions of interexperiment interval.

This experiment closely parallels one of the conditions in a set of studies reported by Shepard (1966), who also had Ss attempt to discriminate new pictures from pictures they had examined during a recognition memory experiment ranging from $2 \mathrm{~h}$ to 120 days before. Perhaps the most important procedural difference from the point of view of comparing results is this: Shepard used a forced choice technique in which $S$ was shown an old and a new picture on each trial and was asked to judge which was the old one. This procedure has the advantage that it avoids the "criterion" or response bias problems associated with the case in which $\mathrm{S}$ is free to respond either "old" or "new" to each stimulus, as he was in the present experiment. Other differences too should be noted; for example, in Shepard's experiment each of the old items of the second experiment had occurred only once (as a member of the inspection series) during the first experiment. Shepard's Ss were self-paced, whereas in the present case the pacing was controlled, the photos being examined at the rate of 1 every $5 \mathrm{sec}$. (It is interesting to note, however, that Ss in the former study spent an average of $5.9 \mathrm{sec}$ on a picture.) Finally, the conditions under which the Ss were initially exposed to the pictures (the original recognition memory experiments) were not identical. In spite of these differences, the results, in terms of per cent correct responses, are in fairly good agreement. In both cases they indicate substantial retention of briefly presented pictorial information over fairly long periods of time.

\section{REFERENCES}

NICKERSON, R. S. Short-term memory for complex meaningful visual configurations: a demonstration of capacity. Canad. J. Psychol., 1965, 19, 155-160.

SHEPARD, R. N. Recognition memory for words, sentences and pictures. $J$. verbal L, earn. verbal Behav., 1967, 6, 156-J 63.

\section{NOTE}

1. This research was performed while the author was a member of the Decision Sciences Laboratory of the Air Force Electronic Systems Division, Air Force Systems Command. Miss Juliana Peterson helped prepare the stimulus materials and collected the data. 